\title{
Influence du climat sur le développement de la population de Varroa jacobsoni Oud dans des colonies d'Apis mellifera iberica (Goetze) dans le sud de l'Espagne
}

\author{
P García-Fernández 1, R Benítez Rodriguez 2, \\ FJ Orantes-Bermejo ${ }^{1}$
}

\begin{abstract}
1 Departamento de Producción Animal, Centro de Investigación y Desarrollo Agrario (CIDA), Consejería de Agricultura y Pesca, Junta de Andalucia, C/Camino de Purchil s/n, 18004 Grenade;

2 Departamento de Parasitología, Facultad de Farmacia, Campus Universitario La Cartuja, Universidad de Granada, 10071 Grenade, Espagne
\end{abstract}

(Reçu le 30 novembre 1994; accepté le 6 mars 1995)

\begin{abstract}
Résumé - Nous avons étudié la dynamique de la population de $V$ jacobsonien Andalousie, dans le sud de l'Espagne, région ou il existe de grands contrastes climatiques. Un échantillonnage mensuel a été effectué, de juillet 1990 à octobre 1992, sans traitement acaricide, sur 26 ruches réparties dans 9 ruchers tout au long du territoire andalou à l'exception d'une ruche située à Cáceres. Ces zones correspondent aux 3 régions climatiques suivantes : méditerranéenne continentale et océanique (MCO), continentale méditerranéenne (CM) et méditerranéenne subtropicale (MS). Le développement des populations d'acariens a été étudié en tenant compte des paramètres suivants : mortalité naturelle (M), taux d'infestation des abeilles adultes (TIA) et taux d'infestation du couvain (TIC). Les taux d'infestation pour les 3 types de climats ont été, respectivement, de 9,$9 ; 4,1$ et 6,1 acariens pour 100 abeilles adultes. De même, les taux d'infestation du couvain ont été de 34,$2 ; 17,8$ et 24,8 acariens pour 100 alvéoles. La période d'infestation maximale a varié en fonction de la région climatique. Les valeurs globales, pour les 3 régions étudiées, ont été de 8,2 acariens pour 100 abeilles et 29,5 acariens pour 100 alvéoles.
\end{abstract}

Varroa jacobsoni / Apis mellifera iberica / dynamique de la population / climat / Espagne

\section{INTRODUCTION}

Varroa jacobsoni Oudemans (1904) est un acarien ectoparasite de l'abeille domestique, qui provoque une maladie parasitaire, la varroose, considérée comme la plus grande menace pour l'abeille dans le monde.

L'action des apiculteurs par la transhumance, l'élevage et la commercialisation des reines a fait que l'aire de distribution 
s'est étendue de l'Asie vers l'Europe et l'Amérique (De Jong et al, 1982b). Depuis l'apparition de $V$ jacobsoni en Espagne en 1985 , les études sur la dynamique des populations d'acariens ont été peu nombreuses. Cependant, il existe des travaux sur l'incidence de cet acarien dans plusieurs régions espagnoles. Puerta et al(1988) soulignent l'importance de la transhumance dans la dispersion de la varroose. Catalayud et Verdu (1992) ont étudié la dynamique des populations dans un rucher de la province de Valencia avec un climat méditerranéen du type Levant-Baléare et ils ont observé une population maximale d'acariens sur les abeilles adultes durant les mois d'avril et mai.

La sensibilité au climat et son influence sur la dynamique de la population de $V$ jacobsoni ont été mises en évidence par plusieurs auteurs (De Jong et al, 1984 ; Ritter et De Jong, 1984 ; Fries et al, 1990 ; Moretto et al, 1991). De même, des études ont été réalisées sur les fluctuations d'autres caractéristiques qui exercent une influence directe sur le cycle biologique de Varroa telles que la période pendant laquelle les cellules de couvain sont ouvertes (Schulz, 1984 ; Moritz et Hänel, 1984 ; Schousboe, 1991), le taux de reproduction de l'acarien (Ifantidis, 1984 ; Puerta et al, 1989) et la fertilité, mesurée par le nombre de femelles non reproductrices (Ruttner et Marks, 1984 ; Camazine, 1986 ; Kulincevic et al, 1988). Fries et al (1994) ont réalisé une synthèse sur le comportement de Varroa dans les différentes sous-espèces d'Apis mellifera $L$ et ils ont proposé une série de modèles théoriques sur la dynamique des populations de l'acarien.

La sensibilité des sous-espèces, races et biotypes d'abeilles face aux acariens a également été analysée. II a été démontré l'existence d'une tolérance aux parasites différente chez les abeilles africanisées et européennes dans des conditions tropicales (Camazine, 1986), et l'existence d'une dif- férence dans la dynamique des populations dans 3 sous-espèces d' $A$ mellifera (Otten, 1988). Par ailleurs, Kulincevic et Rinderer (1988) ont analysé les variations entre les colonies parasitées d'un même biotype d'abeille. La dynamique des populations de $\checkmark$ jacobsoni dépend directement du climat, de l'hôte, des rythmes de développement de l'abeille et de croissance des acariens.

Dans ce travail, nous avons étudié l'influence du climat sur le développement de la population de $V$ jacobsoni pour la sous-espèce $A$ mellifera iberica.

\section{MATÉRIEL ET MÉTHODES}

\section{Localisation des stations d'échantillonnage}

Pour l'échantillonnage, nous avons choisi 9 ruchers situés dans des régions correspondant à 3 zones climatiques différentes (fig 1). Ces stations d'échantillonnage étaient réparties ainsi (d'après Capell Molina, 1981) :

i) La vallée du Guadalquivir, le littoral océanique et l'Estrémadure, qui représentent une zone comportant un climat principalement méditerranéen continental, mais avec une frange côtière ayant un climat méditerranéen océanique ; l'ensemble de cette zone étant considéré comme une seule région climatique (MCO), caractérisée par une température moyenne annuelle de $18^{\circ} \mathrm{C}$ et une humidité moyenne relative assez élevée. Nous y avons installé 12 colonies à 6 emplacements différents.

ii) La zone des plateaux de l'Andalousie orientale et le sillon Intrabétique présente un climat continental méditerranéen (CM) avec une température moyenne annuelle comprise entre $13^{\circ} \mathrm{C}$ et $15^{\circ} \mathrm{C}$ et une variation thermique annuelle assez élevée (de $7^{\circ} \mathrm{C}$ à $20^{\circ} \mathrm{C}$ ). L'humidité moyenne est inférieure à celle de la région climatique précédente et les périodes de gelée sont assez fréquentes. Nous avons échantillonné 8 colonies situées dans 2 emplacements.

iii) La zone de la côte subtropicale méditerranéenne (MS), ayant un climat méditerranéen subtropical, présente de faibles oscillations ther- 


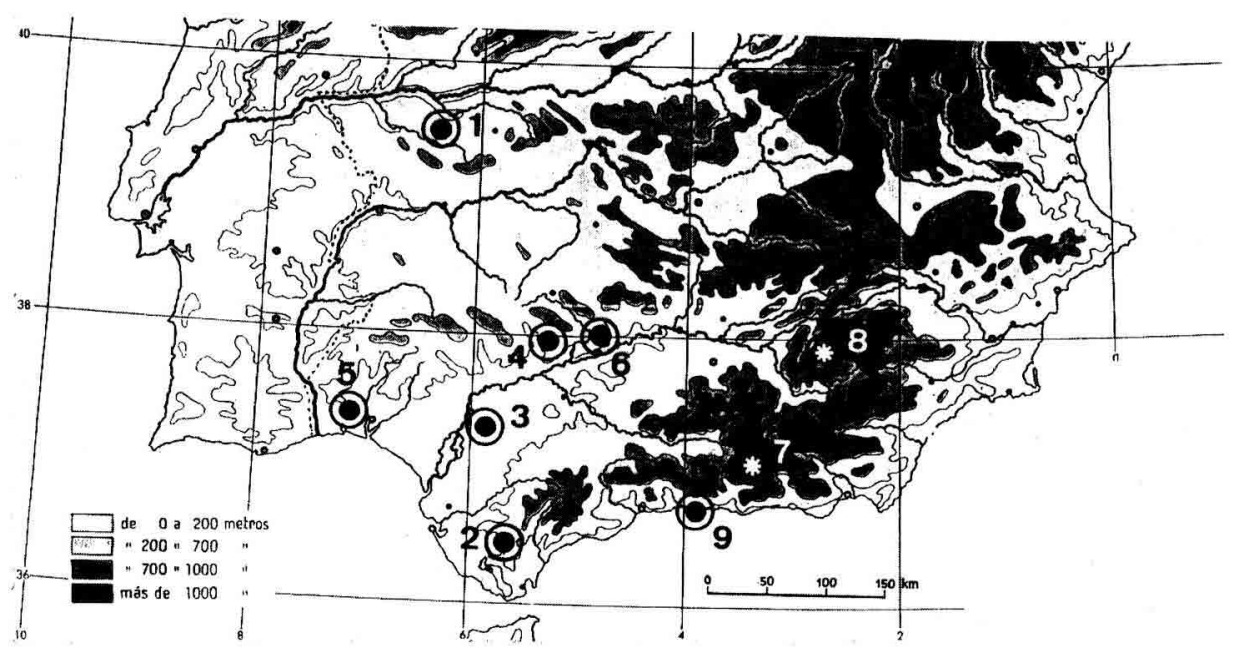

Fig 1. Distribution géographique des stations d'échantillonnage : méditerranéen continental et océanique (MCO) 1 : Cáceres, 2 : Castellar de la Frontera, 3 : Dos Hermanas, 4 : Hornachuelos, 5 : Lepe, 6 : Trassierra ; continental méditerranéen (CM) 7 : Lanjarón, 8 : Vadillo-Cazorla ; méditerranéen suptropical (MS) 9 : Maro-Nerja.

miques, un hiver doux et une température moyenne annuelle de $18^{\circ} \mathrm{C}$ à $19^{\circ} \mathrm{C}$. Dans cette zone, nous avons étudié 6 colonies situées à un même endroit.

\section{Méthodologie}

L'échantillonnage a été réalisé de juillet 1990 à octobre 1992. Le nombre total de ruches étudiées a été de 26, réparties en 9 ruchers situés dans les 3 zones climatiques définies. Chaque rucher comportait 2 ou 3 jeunes colonies qui provenaient d'essaims achetés à des apiculteurs de chacune des zones. Pendant les 3 mois qui ont précédé les échantillonnages, nous avons constaté l'absence de parasitisme par Varroa, et nous avons vérifié, par une étude biométrique, que les abeilles étudiées correspondaient à la sous-espèce $A$ m iberica Goetze (Orantes y García-Férnandez, 1995). Pendant notre étude, les ruches n'ont subi aucun traitement acaricide afin d'étudier la progression naturelle des acariens en fonction des différents climats. Les ruches ont été échantillonnées mensuellement, et, au fur et à mesure qu'elles mouraient, ont été remplacées par de nouvelles colonies

\section{Paramètres étudiés}

\section{Mortalité naturelle (M)}

Nombre d'acariens morts par ruche/mois sur un plancher en carton mince quadrillé et graissé avec de la vaseline.

\section{Taux d'infestation des abeilles adultes (TIA)}

Nous avons défini le TIA comme le taux d'infestation par Varroa des abeilles adultes d'une seule colonie. Tous les mois, ont été échantillonnées de 100 à 250 abeilles par colonie prises au hasard (modifié de Papas et Thrasyvoulou, 1986). Nous avons suivi la méthode d'étude décrite par Ritter (1981) et De Jong et al (1982a)

\section{Taux d'infestation du couvain (TIC)}

Dans chacune des ruches, nous avons prélevé mensuellement, au hasard, une section de couvain (operculé et non operculé), mesurant environ 15 sur $5 \mathrm{~cm}$. Le TIC est le pourcentage de Varroa 
qui se trouvent dans 100 alvéoles (operculées et non operculées).

\section{Méthodes statistiques}

Parmi les variables de la population considérées dans l'étude de l'évolution du parasitisme, le TIA et le TIC ont été utilisés pour vérifier s'il y avait des différences entre les 3 zones climatiques. Nous avons réalisé une analyse de variance avec le total de chacun des taux d'infestation, tout en analysant aussi les différentes paires possibles qui peuvent être formées avec ces 3 groupes climatiques. Pour ce faire, la méthode que nous avons appliquée est celle de Scheffé pour $\alpha=0,01$. Pour obtenir une variance homogène, les données des variables TIA et TIC ont été transformées en appliquant l'expression [y= $\operatorname{arctang}(x)]$. Pour les analyses, nous nous sommes servis d'un progiciel statistique SPSS/PC (Nie et al, 1983).

\section{RÉSULTATS}

\section{Effets du climat sur le développement de V jacobsoni}

Pour les différents climats considérés, nous avons représenté l'évolution des valeurs moyennes de M, TIA et TIC au cours de l'échantillonnage de toutes les ruches groupées en fonction du climat auquel elles appartenaient, et cela si l'homogénéité des données nous le permettait (fig 2). Les moyennes des TIC dans les groupes climatiques $\mathrm{CM}, \mathrm{MCO}$, et MS ont été respectivement de $17,8 \pm 5,6 ; 34,2 \pm 7,4$ et $24,8 \pm$ 9,1 acariens pour 100 alvéoles. Nous avons aussi observé une différence importante entre elles ( $F=15,2, p<0,001)$ au cours de l'analyse 2 à 2 ; le niveau de signification désiré n'a été obtenu que par la paire formée par les groupes climatiques CM-MCO et CM-MS. Les moyennes des TIA dans les groupes climatiques $\mathrm{CM}, \mathrm{MCO}$ et MS ont été respectivement de $4,1 \pm 0,5 ; 9,9 \pm 1,7$ et $6,1 \pm 1,4$ acariens pour 100 abeilles et nous avons de même observé une différence importante entre elles $(F=7,52, p<$ 0,001 ) au cours de l'analyse comparative des paires 2 à 2 . Le couple formé par les groupes climatiques CM-MCO est le seul qui ait obtenu la signification désirée (tableau I).

Pour l'évolution de la dynamique de la population en climat CM, les maxima d'infestation commencent à partir de février-mars, et les niveaux moyens d'infestation sont

Tableau I. Valeurs totales de mortalité $(M)$ et valeurs moyennes \pm SE du taux d'infestation des abeilles adultes (TIA) et du taux d'infestation du couvain (TIC) sous les 3 climats considérés pendant la période étudiée.

\begin{tabular}{|c|c|c|c|c|}
\hline & $\begin{array}{l}\text { Climat continental } \\
\text { méditerranéen }\end{array}$ & $\begin{array}{l}\text { Climat méditérranéen } \\
\text { continental et océanique }\end{array}$ & $\begin{array}{c}\text { Climat méditerranéen } \\
\text { subtropical }\end{array}$ & Total \\
\hline$M$ & $\begin{array}{l}10723 \\
n=69\end{array}$ & $\begin{array}{c}7940 \\
n=126\end{array}$ & $\begin{array}{l}16987 \\
n=36\end{array}$ & $\begin{array}{l}35650 \\
n=231\end{array}$ \\
\hline TIA (\%)a & $\begin{array}{c}4,1 \pm 0,5 \\
n=69\end{array}$ & $\begin{array}{c}9,9 \pm 1,7 \\
n=130\end{array}$ & $\begin{array}{c}6,1 \pm 1,4 \\
n=36\end{array}$ & $\begin{array}{c}8,2 \pm 1,2 \\
n=235\end{array}$ \\
\hline $\operatorname{TIC}(\%)^{b}$ & $\begin{array}{c}17,8 \pm 5,6 \\
n=49\end{array}$ & $\begin{array}{c}34,2 \pm 7,4 \\
n=73\end{array}$ & $\begin{array}{c}24,8 \pm 9,1 \\
n=26\end{array}$ & $\begin{array}{c}29,5 \pm 7,6 \\
n=148\end{array}$ \\
\hline
\end{tabular}

$n=$ effectif ; $M=$ total d'acariens morts sur le plancher pendant la période d'échantillonnage ; a moyennes avec différences significatives $(\mathrm{F}=7,52 ; p<0,001)$; $\mathrm{b}$ moyennes avec différences significatives $(\mathrm{F}=15,2 ; p<0,001)$. 

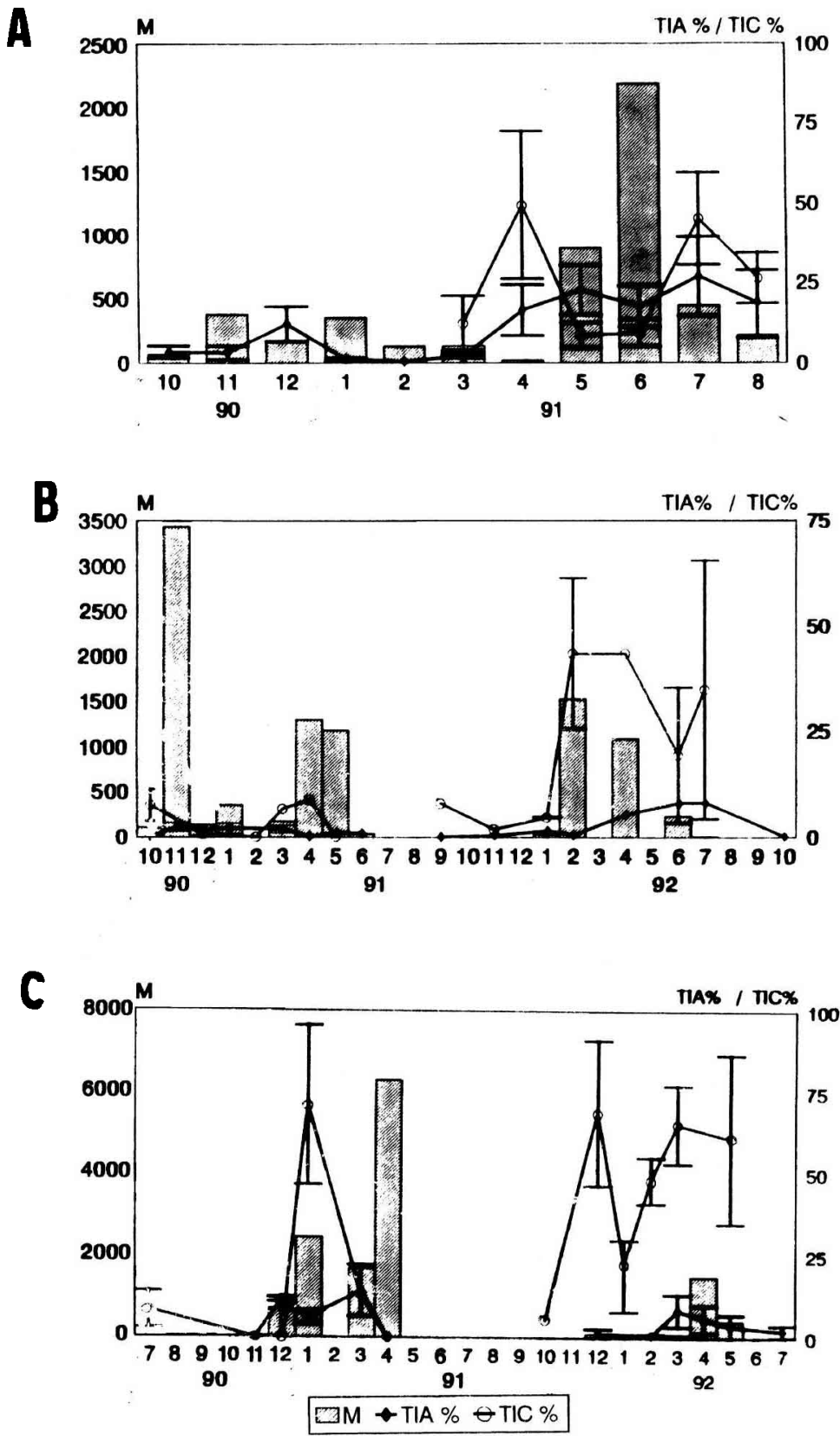

Fig 2. Dynamique des populations de Varroa jacobsoni sur Apis mellifera iberica dans le sud de l'Espagne sous les 3 climats considérés. A : méditerranéen continental et océanique (MCO) ; $\mathbf{B}$ : continental méditerranéen (CM) ; $\mathbf{C}$ : méditerranéen suptropical (MS). $M=$ Barré, total d'acariens morts sur le plancher des ruches/mois. TIA = taux d'infestation des abeilles adultes. $T I C=$ taux d'infestation du couvain. Les données des TIA et TIC sont exprimées en moyennes \pm SE. 
faibles ( $\mathrm{TIA}=4,1 ; \mathrm{TIC}=17,8$ ), tandis qu'en climat MCO les maxima apparaissent au mois de mars et les niveaux d'infestation moyens sont plus du double que pour le climat précédent $(\mathrm{TIA}=9,9 ; \mathrm{TIC}=34,2)$ (fig 2 , tableau I).

Le climat joue un rôle décisif sur le TIA et le TIC, surtout dans les groupes climatiques $\mathrm{CM}$ et $\mathrm{MCO}$, tandis que l'effet du groupe climatique MS sur l'acarien se situe entre celui des 2 autres climats. Ces climats correspondent à des écosytèmes très différents. Le climat $\mathrm{CM}$ comprend les zones de moyenne et haute montagne du système Pénibétique, tandis que le climat $\mathrm{CMO}$ correspond à la vallée du Guadalquivir et à son aire d'influence, et comprend aussi la partie nord de l'Andalousie occidentale et l'Estrémadure qui se caractérisent par des zones de basse montagne et des pâturages.

D'après ce que nous avons observé, nous pouvons affirmer qu'en Andalousie $V$ jacobsoni provoque la mortalité des ruches dans les 8 à 15 mois après le début de l'infestation.

\section{DISCUSSION}

\section{Évolution de la dynamique des populations de Varroa sous les différents climats}

La dynamique des populations de $V$ jacobsoni est fortement liée au développement biologique de son hôte. En Andalousie, il existe une grande diversité d'écosystèmes et de climats, ce qui fait que les cycles annuels d'activité des abeilles sont très différents, et ceci conditionne la dynamique de développement des acariens.

Dans cette région, Varroa a suivi 2 modèles de croissance différents, un modèle bimodal qui se manifeste dans la majorité des zones étudiées et l'autre modèle, du type exponentiel, localisé à la côte subtropicale. En ce qui concerne le premier modèle, on a observé une variation de la période d'infestation maximale (fig 2). Dans le climat $\mathrm{MCO}$, caractéristique de l'Andalousie occidentale, la population d'acariens atteint sa croissance maximale de 1 à 3 mois plus tard par rapport à l'Andalousie orientale. Ce retard de la population maximale des acariens pourrait être lié à certains facteurs comme le climat et les périodes de floraison (phénologie).

En général, dans le sud de l'Espagne, le cycle biologique de $V$ jacobsoni est plus précoce qu'en Europe continentale. Dans l'étude sur la dynamique des populations de Varroa réalisée sur un rucher de la province de Valencia, Calatayud et Verdu (1992) ont observé des maxima d'infestation (TIA) pendant les mois d'avril-mai. Ces résultats sont semblables à ceux que nous avons obtenus pour le climat $\mathrm{MCO}$, étant donné que les paramètres climatiques sont sensiblement identiques. Dans notre étude, nous avons obtenu les populations maximales de mars à juin, tandis qu'en Allemagne Ritter et al (1984) et Moritz et Mautz (1990) ont observé les maxima aux mois de juillet et août.

Les différences observées dans la dynamique des populations de Varroa pourraient être dues à la floraison précoce des plantes nactarifères en Andalousie orientale (romarins, amandiers, genêts, certains thyms, etc) (Ortega, 1986), qui entraîne la précocité du cycle biologique des Varroa. Les basses températures peuvent également modifier la période de développement du couvain operculé (Woyke, 1987), provoquant ainsi l'augmentation du nombre de cycles de reproduction de l'acarien et, par conséquent, de la croissance de la population, telle qu'on l'a vérifiée dans les zones les plus froides. La température et la floraison précoce peuvent donc provoquer, dans les zones froides de l'Andalousie orientale, l'apparition des maxima pendant le mois de février, quand 
les périodes de gelées sont encore fréquentes, ce qui provoque l'affaiblissement irréversible des colonies. En revanche, en Andalousie occidentale et en Estrémadure, où les températures sont plus douces en hiver, le développement du parasite est constant jusqu'à la fin du mois de juin, moment où se produit le collapsus des colonies. Par ailleurs, les niveaux moyens d'infestation détectés ont été le double pour le climat MCO que pour le climat CM (tableau I et fig 2).

D'après les résultats d'une étude réalisée au Brésil sous 3 climats différents, les taux d'infestation ont été plus élevés en climat très froid, et l'on a constaté que l'évolution du parasitisme par Varroa dépend plus du climat que des caractères génétiques de l'abeille (Moretto et al, 1991).

En climat subtropical, nous avons constaté des taux d'infestation élévés dans le couvain, avec des maxima de développement de Varroa pendant les mois de janvier et février ; généralement, les colonies disparaissaient peu après ce maximum. Ce développement des populations de Varroa est accéléré en climat subtropical qui permet la floraison pendant toute l'année, qu'il s'agisse de végétation autochtone, de cultures subtropicales ou de cultures en serres. La climatologie et la phénologie sont donc 2 facteurs qui peuvent contrôler la dynamique des populations de $V$ jacobsoni. Pour cette étude, nous n'avons tenu compte ni des différents comportements des acariens face aux abeilles, ni de l'existence des différentes caractéristiques génétiques des hôtes dans les 3 régions climatiques analysées. Grâce à la sélection génétique, on espère potentialiser certains facteurs biologiques et comportementaux des abeilles, afin d'obtenir des variétés plus résistantes au Varroa. En 1987, Kulincevic et al (1992) ont commencé à sélectionner en ex-Yougoslavie 2 lignées d'abeilles, l'une de faible résistance et l'autre de résistance élevée à Varroa.

\section{Analyse des paramètres considérés de la population}

Les valeurs de la mortalité de Varroa ne sont pas corrélés à l'état d'infestation de la colonie à un moment précis (Rademacher, 1985). Dans notre étude, aucun rapport n'a été observé entre la mortalité ( $M$ ), les TIA et les TIC ; cependant, le TIC est le paramètre qui reflète le mieux le degré d'infestation de la colonie.

\section{REMERCIEMENTS}

Le présent travail a été financé par l'Instituto Nacional de Investigaciones Agrarias, Projet INIA $n^{\circ} 9108$. Nous remercions $F$ Martinez et M Valenzuela, du Centro de Capacitación y Experimentación Forestal de Cazorla (Jaén), le Dr F Puerta de la Cátedra de Biología Aplicada de la Facultad Veterinaria de Córdoba, le Dr JM Alonso du Dpto de Sanidad Animal, S Sayadí, du Dpto de Economia Agraria (Universidad de Cáceres), I Martín Bravo, technicienne du laboratoire du Dpto de Producción Animal (CIDA, Grenade) et les apiculteurs qui nous ont aidés dans la réalisation du travail de terrain.

\section{Summary - Influence of climate on the evolution of the population dynamics of the Varroa mite on honeybees in the south of Spain. The population dynamics of the mite Varroa jacobsoni were studied in colonies of honeybees (Apis mellifera iber- ica) in the south of Spain (Andalusia), a region of wide climatic contrasts.}

Between July 1990 and October 1992, samples and data were collected monthly from 26 beehives untreated for parasites. The colonies were located in 9 apiaries in 3 areas with different climates (fig 1). The development of the mite population was measured by determining the infestation rate of Varroa mite in adult bee, in brood cells and the mortality per month. In the 3 different climatic areas studied: mediter- 
ranean, continental and oceanic (MCO), continental mediterranean (CM) and mediterranean subtropical (MS), the mean infestation rates were $9.9,4.1$ and 6.1 mites per 100 adult bees, respectively. The mean number of mites per 100 brood cells was $34.2,17.8$, and 24.8 . The overall mean of mite infestation in the study was 8.2 mites per 100 bees, ie 29.5 mites per 100 brood cells (table I). Monthly differences in maximal infestation were observed in the areas studied (fig 2).

In these areas $V$ jacobsoni speeds up its biological cycle compared with the descriptions reported by other authors in continental Europe. $V$ jacobsoni reaches its infective maxima earlier in the sampling stations with colder climates (CM) than in those with hotter climates (CMO). The results of our study confirm the observation of Woyke (1987) that $V$ jacobsoni stays longer in sealed cells when climates are colder. The parasitic mite thus completes more reproductive cycles in the same period of time, speeds up the population growth.

Milder climates favour global infestation rates to be higher in hotter areas (CMO). This totally different behaviour can be observed on the mediterranean coast of the Iberian Peninsula (MS). The effects of the climate in this area, together with the occurrence of efflorescence (autochtonic, from subtropical cultivations or from greenhouse cultivations) throughout the year, cause fast development of the parasitic mite leading to the death of honeybee colonies within a few months. Our study shows that the phenology and climate conditions of a region are 2 main factors which affect and modify $\checkmark$ jacobsoni population dynamics.

Varroa jacobsoni / Apis mellifera iberica / dynamic population / climate / Spain

\footnotetext{
Zusammenfassung - Einfluß des Klimas auf die Populationsdynamik der
}

Milbe Varroa jacobsoni Oud in Völkern von Apis mellifera iberica (Goetze) in Südspanien. Die Populationsdynamik der Milbe Varroa jacobsoni in Bienenvölkern (Apis mellifera iberica) wurde in einem Gebiet untersucht, das große klimatische Unterschiede aufweist. Zwischen Juli 1990 und Oktober 1992 wurden von 26 nicht mit Varroamitteln behandelten und auf 9 Bienenstände ( $A b b$ 1) mit unterschiedlichen Klimabedingungen verteilten Völkern jeden Monat Proben genommen. Zur Messung der Entwicklung der Milbenpopulation wurde die Infektionsrate der Milben auf adulten Bienen, in den Brutzellen und der natürliche Totenfall bestimmt. Drei unterschiedliche Klimabereiche wurden untersucht: mediterran-continentales Meeresklima (MCO), mediterran-continentales (CM) und mediterran-subtropisches Klima (MS). Die durchschnittlichen Befallsraten betrugen 9,9 Milben, 4,1 bzw 6,1 Milben pro 100 adulten Bienen und $34,2 / 17,8$ und 24,8 pro 100 Brutzellen. Der Gesamtmilbenbefall lag somit bei dieser Untersuchung bei 8,2 Milben pro 100 Bienen und 29,5 Milben pro 100 Brutzellen (Tabelle 1). In den untersuchten Gebieten ergaben sich Unterschiede zwischen den Monaten mit der höchsten Infektionsrate (Abb 2).

Insgesamt ist der biologische Zyklus von $V$ jacobsoni in unserem Beobachtungsgebiet beschleunigt, wenn man die Werte mit denen anderer Autoren aus gemäßigten Klimazonen vergleicht. $V$ jacobsoni erreicht das Populationsmaximum auf dem Versuchsstand mit kälterem Klima (CM) früher als auf dem mit dem wärmeren Klima (CMO). Diese Ergebnisse bestätigen die Hypothese von Woyke (1987). Er beobachtete, daß Milben kürzere Zeit außerhalb der verdeckelten Brutzellen bleiben, wenn das Klima kälter ist. Die parasitische Milbe vollendet dadurch mehr reproduktive Zyklen in der gleichen Zeitspanne und beschleunigt dadurch das Populationswachstum. Das frühe Auftreten bzw die frühe Blüte von 
Nektarpflanzen im kälteren Gebiet von Andalusien trägt ebenfalls zu dieser Wirkung auf die Populationsdynamik bei. Auf der anderen Seite begünstigt ein mildes Klima die Befallsrate, sodaß sie in wärmeren Gebieten (CMO) insgesamt höher ist. Ein völlig unterschiedliches Verhalten kann dagegen an der Mittelmeerküste der Iberischen Halbinsel (MS) beobachtet werden. Das Klima in diesem Gebiet bewirkt gemeinsam mit der ganzjährigen Blütezeit von sowohl autochtonen Pflanzen als auch von Pflanzen aus subtropischer Kultivierung oder aus Kulturen im Gewächshaus eine schnelle Entwicklung des Parasiten und führt durch eine exponentiale Vermehrungsrate in wenigen Monaten zum Tod des Bienenvolks.

Unsere Untersuchungen zeigen, daß die Phänologie und die klimatischen Bedingungen einer Region 2 Hauptfaktoren bilden, die auf die Entwicklung von $V$ jacobsoni einen Einfluß haben und die die Populationsdynamik modifizieren.

\section{Varroa jacobsoni / Apis mellifera iberica / Populationsdynamik / Klima / Spanien}

\section{RÉFÉRENCES}

Calatayud F, Verdu MJ (1992) Evolución anual de parámetros poblacionales de colonias de Apis mellifera $\mathrm{L}$ (Hymenoptera: Apidae), parasitadas por Varroa jacobsoni Oud (Mesostigmata: Varroidae). Boletin de Sanidad Vegetal Plagas 18, 777-788

Camazine S (1986) Differential reproduction of the mite Varroa jacobsoni (Mesostigmata: Varroidae), on Africanized and European honey bees (Hymenoptera: Apidae). Ann Entomol Soc Am 79, 801-803

Capell Molina JJ (1981) Los climas de España. Editorial Oikos-tau. SA, Primera edición, $429 p$

De Jong D, De Jong P, Gonçalves LS (1982a) Weight loss and another damage to developing worker honey bees (Apis mellifera) due to infestation with Varroa jacobsoni. J Api Res 21, 165-167

De Jong D, Gonçalves LS, Morse RA (1984) Dependence on climate of the virulence of $V$ jacobsoni. Bee World 65, 117-121

De Jong D, Roger A, Morse G, Eickwort C (1982b) Mite pest of honeybees. Annu Rev Entomol 27, 229-252
Fries I, Aarhus A, Hamsem H, Korpela S (1990) Development of early infectations by $V$ jacobsoni in honey bee colonies in cold climates. Exp Appl Acarol 11, 205-214

Ifantidis MD (1984) Parameters of the population dynamics of the Varroa mite on honey bees. J Apic Res $23,227-233$

Kulincevic JM, Rinderer TE (1988) Breeding honey bees for resistence to Varroa jacobsoni. Analysis of the mite population dynamics. In : Africanized Honey Bees an Bee Mites (GR Needham et al, eds), Chichester, 434-443

Kulincevic, JM, Rinderer TE, Urosevic DJ (1988). Seasonality an colony variation of reproducing and nonreproducing Varroa jacobsoni females in western honey bee (Apis mellifera) worker brood. Apidologie, 20, 173-180

Kulincevic JM, Rinderer TE, Mladjan VJ, Buco SM (1992) Five years of bidirectional genetic selection for honeybees resistant and susceptible to $V$ jacobsoni. Apidologie 5, 443-452

Moretto G, Gonçalves LS, De Jong D, Bichuette MT (1991) The effects of climate and bee race on $V$ jacobsoni Oud infections in Brasil. Apidologie 22, 197-203.

Moritz RFA, Hänel H (1984) Restricted development of the parasitic mite Varroa jacobsoni Oud in the cape honey bees Apis mellifera capensis Esch. Z Angew Entomol 97, 91-95

Moritz RFA, Mautz D (1990) Development of Varroa jacobsoni in colonies of Apis mellifera capensis and Apis mellifera carnica. Apidologie 21, 53-58

$\mathrm{Nie} \mathrm{NH}$, Hol CH , Jenkins JG, Steinbrenner K, Bent DH (1983) SPSSX Statistical Package for the Social Sciences. New York, McGraw-Hill

Orantes Bermejo FJ, Garcia Fernandez P (1995) Morphological variability of Apis mellifera iberica Goetze (1964) in different apiaries of southern Spain. J Apic Res 34, 23-30

Ortega JL (1986) Flora de interés apícola y polinización de cultivos. Mundi-Prensa, $149 \mathrm{p}$

Otten C (1988) A comparation of Varroa jacobsoni populations dynamics in different subspecies of $A$ mellifera. Proc of a meeting of the EC-Experts'Group, Udine, Italy, 1988. Present Status of Varroosis in Europe and Progress in the Varroa Mite Control $(R$ Cavalloro, ed), 101-106, Comiss Europ Commun Joint Res Centre 1-21020 Ispra

Papas N, Thrasyvoulou A (1986) Searching for an accurate method to evaluate the degree of Varroa infestation in honey bees colonies. Proceedings of a meeting of the EC-Experts Group, European Research on Varroosis Control, Bad Homburg, Oct 1986 (R Cavarollo, ed), 85-86, Comiss Europ Commun Joint Res Centre, 1-21020 Ispra

Puerta F, Flores JM, Bustos M, Padilla F, Fernandez FJ (1988) Características epizoóticas de la parasi- 
tosis por $V$ jacobsoni Oud en colmenares de la provincia de Almeria. Revista lbérica de Parasitologia 48, 195-202

Puerta F, Castellano A, Bustos M, Pellin P, Flores JM, Padilla F (1989) Variabilidad en la tasa reproductiva de $V$ jacobsoni en colmenas de Apis mellifera iberica. Revista lbérica de Parasitología 49, 381 386

Rademacher E, Geiseler E (1985) Varroa disease of honeybees: history, diagnosis, therapy (SJ Schelzky, ed), Berlin, $104 \mathrm{p}$

Ritter W (1981) Varroa disease of the honeybees Apis mellifera. Bee World 62, 141-153.

Ritter W, De Jong D (1984) Reproduction of $V$ jacobsoni Oud in Europe, the Middle East and tropical South America. Z Angew Entomol 98, 55-57
Ritter W, Leclercq E, Koch W (1984) Observations on and Varroa jacobsoni mite populations in infested honey bees colonies. Apidologie 15, 389-400

Ruttner F, Marks GH (1984) Beobachtungen über eine mögliche Anpassung von Varroa jacobsoni an Apis mellifera $L$ in Uruguay. Apidologie 15, 43-62

Schousboe C (1991) Varroasis y desarrollo larvario de las abejas. Vida Apícola 45, 36-45

Schultz A (1984) Reproduktion und Populationsentwicklung der parasitischen Milbe Varroa jacobsoni Oud in Abhängigkeit vom Brutzyklus ihres Wirtes Apis mellifera L (I Teil). Apidologie 15, 401-420

Woyke J (1987) Population dynamics parameters of Tropilaelaps clareae in comparison with those Varroa jacobsoni mites on honey bees. J Apic Res 26 , 196-202 